REVISTA ECONOMÍA

Vol. 67, N. ${ }^{\circ}$ Io6 (noviembre 2015), I45-I63

\title{
MUJERES Y TRABAJO EN LUGARES DE EXTRACCIÓN Y REFINAMIENTO PETROLERO EN ECUADOR
}

\author{
IVETTE VALLEJO, FERNANDO GARCÍA, CRISTINA CIELO \\ Facultad Latinoamericana de Ciencias Sociales, sede Ecuador
}

Recepción manuscrito: 4 de agosto de 2015

Aceptación versión final: 30 de octubre de 2015

\begin{abstract}
RESUMEN El artículo aborda la situación de las mujeres en zonas del circuito petrolero: en la ciudad de Esmeraldas, donde hay una larga historia de incorporación a la industria petrolera, y en zonas de la Amazonía norte del país (Sucumbíos) en las que proyectos de desarrollo local acompañan la extracción del petróleo. Se sintetizan y comparan las situaciones de trabajo de las mujeres en Esmeraldas y en Sucumbíos en base a una investigación efectuada con metodologías cualitativas y revisión documental. Asimismo se profundizan en las formas de exclusión social que se generan en la medida en que los territorios donde habitan están engranados a la acumulación de capital y al flujo de commodities para el mercado global.
\end{abstract}

palabRAS CLAVE Trabajo, exclusión, informalidad, cuidado, extractivismo.

ABSTRACT This article addresses the situation of women in oil circuit areas: in Esmeraldas city, where there is a long story of their incorporation to the oil industry, and in the northern Amazon area in Ecuador (Sucumbíos), where local development projects accompany oil extraction. We summarize and compare women's work situation in Esmeraldas and Sucumbíos based on some research carried out with qualitative methodologies and a documentary revision. Likewise, we analyze the social exclusion ways that are generated to the extent that the territories where they dwell are linked to the accumulation of capital and the flow of commodities for the global market.

KEYWORDS Work, exclusion, informality, care, extractivismo.

JEL CODES J16, L72.

\section{INTRODUCCIÓN}

Desde los inicios del auge extractivo en los setenta, la economía ecuatoriana se ha sustentado sobre el extractivismo petrolero. ${ }^{1}$ Pese a su ciclicidad y a la contracción económica, crisis económica y desbalance presupuestario ${ }^{2}$ generado por la caída de los precios del barril de petróleo en distintas coyunturas mundiales, se ha continuado profundizando la matriz extractiva en el país.

El peso de las exportaciones primarias y petroleras durante la Revolución Ciudadana aumentó, en detrimento de las exportaciones no petroleras. Así, si al 2007 las exportaciones 
petroleras en millones de dólares, eran de 8328 ( $58 \%$ del total de exportaciones) y las exportaciones no petroleras eran al 2007 de 5993 (42\% del total de exportaciones); al 2013 las exportaciones petroleras eran de 14.108 (56,5\% del total de exportaciones) y las no petroleras de 10.850 (43,5\% del total), (Caria y Domínguez, 2014, p. 149). ${ }^{3}$ Si bien el gobierno de la Revolución Ciudadana en los últimos años ha puesto énfasis en que se potenciaría el cambio en la matriz productiva, ${ }^{4}$ no consiguió diversificar la estructura productiva del país, ni avanzar la sustitución selectiva de importaciones. En los últimos ocho años, el petróleo ha sustentado la base económica para la implementación del Plan Nacional del Buen Vivir en sus distintas versiones (2009-2013 y 2013-2017), con una cantidad mayor de ingresos recibidos por exportaciones petroleras que en cualquier otro gobierno anterior manteniéndose la esencia extractivista (Acosta, 2012; 2013).

El extractivismo petrolero se ha profundizado integrando nuevos territorios para campos y bloques petroleros en la Amazonía norte y otras zonas de la Amazonía, ${ }^{5}$ así como desde el 2012 se ha intentado ampliar las fronteras extractivas hacia el suroriente, con la XI Ronda. ${ }^{6}$ Esto acelera formas de «acumulación por desposesión» (Harvey, 2004) y de "cercamiento de los bienes comunes naturales» (Harvey, 2014, p. 245) que afecta a comunidades locales, en gran mayoría indígenas, a la vez que trastoca las economías locales, la autonomía de las mujeres y sus prácticas de aprovisionamiento y sustentabilidad. En la misma dirección de afianzar el extractivismo, la refinería en la ciudad de Esmeraldas ha sido repotenciada para el procesamiento de mayores volúmenes de crudo, ${ }^{8}$ lo que igualmente impone sufrimiento ambiental en la población, en su mayor parte afrodescendiente y con especial carga de costes en el trabajo de las mujeres.

En este marco, si bien el Estado ha redirigido mayor inversión de infraestructura de servicios en lugares aledaños a sitios estratégicos donde se realizan actividades extractivas, con el ofrecimiento de la redistribución de la renta petrolera, los lugares donde se refina y se extrae el petróleo no son sitios donde existan condiciones de bienestar. Las mujeres en estos lugares están situadas en economías no formales, en el sector informal en Esmeraldas y en trabajos de agricultura, silvicultura y pesca en Sucumbíos. En ambos casos, estas inversiones estatales han significado una mayor integración de las poblaciones locales a circuitos de consumo de bienes y servicios.

Desde que empezaron a funcionar en Esmeraldas la refinería y TermoEsmeraldas se han direccionado a políticas de compensación, denominadas contemporáneamente de inclusión social. Es así como en los barrios del sur se han ejecutado estudios de agua potable, obras como alcantarillado, asfaltado, ampliación y repotenciación del sistema de agua, subcentros de salud; estas inversiones se han incrementado con las políticas redistributivas de la Revolución Ciudadana, con especial atención a la reconstrucción de centros educativos y el establecimiento de Unidades Educativas del Milenio. Pese a ello, muchos de los barrios continúan sin alcantarillado, o con uno colapsado; sus calles en gran parte de los barrios aún no están asfaltadas y carecen de agua. Con más de cuarenta años de funcionamiento de la refinería, la población esmeraldeña no se beneficia precisamente con fuentes de empleo remunerado relacionados con la actividad de refinamiento de petróleo u otras conexas. Una gran mayoría de la población afroesmeraldeña, principalmente las mujeres, se insertan en actividades dentro del sector 
informal de la economía, sin beneficiarse de la localización de Esmeraldas dentro del circuito petrolero. La refinería ha generado en la población de Esmeraldas impactos ambientales y de salubridad más que beneficios económicos e inclusión social, pese a la mayor inversión que ha habido en los últimos años en obra física e infraestructura.

Sucumbíos también ha experimentado una importante inversión de la renta petrolera beneficiándose de una de las acciones más emblemáticas del gobierno en zonas de interés estratégico: la construcción de Ciudades del Milenio. Hay dos Ciudades del Milenio ya construidas en la Amazonía norte en la provincia de Sucumbíos y una tercera en construcción en la misma provincia. A partir de negociaciones entre sus comunidades y el gobierno, en Pañacocha (en el cantón Shushufindi) y Playas de Cuyabeno se construyeron Ciudades del Milenio con acceso a infraestructura de servicios y tecnologías, además de vivienda y Unidades Educativas del Milenio. Estos proyectos de desarrollo local se construyen a manera de compensación por permitir el inicio de actividades extractivas en sus territorios, sobre los cuales se superpuso el bloque 12 (Edén-Yuturi) operado por la estatal PetroAmazonas. ${ }^{9}$ Una de las principales expectativas de la población fue la generación de fuentes de empleo, tal como lo fue en Esmeraldas con el establecimiento de la refinería. Si las mujeres esmeraldeñas se han incorporado en grandes números al trabajo informal, veremos cómo en Sucumbíos la incorporación de mano de obra masculina de manera coyuntural y no sostenida a las actividades extractivas ha generado mayor carga de trabajo para el aprovisionamiento familiar (en labores agrícolas) en las adultas mujeres y una pérdida de autonomía en el caso de las mujeres jóvenes.

Lo que se evidencia en ambos casos es los efectos contradictorios de la incorporación de las mujeres y las economías locales dentro de la economía monetizada global. Como se observa en el caso de las Ciudades del Milenio, el extractivismo acelera transformaciones conducentes a la incorporación y dependencia en la economía monetaria, integrando a poblaciones locales en una mayor mercantilización de la vida económica. Federici (2004) demuestra que la creación de condiciones materiales para un nuevo régimen monetario, en el que solo la producción para el mercado es considerada como actividad creadora de valor, tuvo lugar con la acumulación originaria del capital. Pero sus argumentos, que demuestran la consecuente desvalorización económica de las actividades reproductivas y de cuidado, bien pueden ser extrapolados a la acumulación en la fase ampliada del capital que integra a nuevos territorios y poblaciones. La importancia económica de la reproducción de la mano de obra llevada a cabo en el hogar y su función en la acumulación del capital, se hacen invisibles.

En zonas extractivas, la economía tiende a monetizarse, lo que siguiendo a Federici (2004), redefine la posición de las mujeres en la sociedad y en relación a los hombres. La división sexual del trabajo que aparece tiende a sujetar a las mujeres al trabajo reproductivo y aumenta su dependencia respecto de los hombres, permitiendo al Estado y a los empleadores usar el salario masculino como instrumento para gobernar el trabajo de las mujeres. De esta manera, «la separación de la producción de mercancías de la reproducción de la fuerza de trabajo hace posible el desarrollo de un uso específicamente capitalista del salario y de los mercados como medios para la acumulación de trabajo no remunerado», (Federici, 2004, p. 112). En una sociedad cada vez más dependiente de la economía del mercado, las mujeres sin acceso a los salarios, pueden ser forzadas «a la condición de una pobreza crónica, la dependencia económica 
y la invisibilidad como trabajadoras», (Federici, 2004, p. 113). Esto se observa con una intensidad brutal en el caso de Esmeraldas.

Tanto las mujeres afrodescendientes en Esmeraldas, como las kichwas y mestizas en Sucumbíos realizan trabajo reproductivo, con lo que nos referiremos al cuidado de los niños, las familias y sus ambientes, $\mathrm{y}$ «todo el trabajo involucrado con la producción de las condiciones de producción, incluyendo la naturaleza en sí misma», (Turner y Brownhill, 2006, p. 89). Se trata de mujeres que no son proletarias, tampoco «trabajadoras libres», son «trabajadoras no-asalariadas, que son la base fundante de la acumulación y el crecimiento», (Turner y Brownhill, 2006, p. 88). Son las reales productoras que crean las condiciones generales para la reproducción social, puesto que el capitalismo en su dinámica depende y se aprovecha de las trabajadoras no asalariadas del mundo. El extractivismo como forma de acumulación capitalista, reduce la autonomía de las poblaciones campesinas, indígenas y afrodescendientes, dinámica en la que las mujeres quedan insertas sin mayor asidero y limitadas en poder; lo que ocurre cuando en la contemporaneidad se reproduce una agresiva incorporación de comunidades y poblaciones, otrora sustentables y autónomas, a la economía de mercado. En contextos extractivos, las mujeres no son integradas como trabajadoras proletarizadas y, potencialmente, como vemos en la politización antiextractiva de mujeres en el suroriente amazónico, podrían tener mayor interés por prevenir la commoditzación capitalista de las relaciones comunales, del ambiente, de sus espacios públicos y comunales frente a los encerramientos (enclosures) que implica una mayor integración al circuito petrolero.

\section{MUJERES AFRODESCENDIENTES Y TRABAJO EN ESMERALDAS}

Una precarización extrema de la situación de las mujeres se ve en Esmeraldas, donde el pueblo afrodescendiente lidia cotidianamente con la discriminación laboral que se expresa como negación a la ciudadanía históricamente racializada. La discriminación laboral opera desde muchas dimensiones, en especial cuando el empleador ejerce el poder y la dominación no solo sobre la fuerza laboral, alcanzando su máximo nivel de explotación y plusvalía, sino cuando la acción discriminante del empleador atenta sobre el derecho del trabajo en condición de dignidad y plena satisfacción por parte del trabajador. En este sentido, la operación de la discriminación laboral va en contra de los derechos de la igualdad al trabajo y a la no discriminación consagrados tanto en la Constitución de 2008 (arts. 1, 3, 11), como en los instrumentos internacionales de derechos humanos, en especial el Convenio 111 de la ort y la Convención contra la Discriminación Racial y la Declaración de Durban de 2001.

Se observa la discriminación laboral que sufren los afrodesciendientes ecuatorianos en la ciudad de Esmeraldas, donde viven 86.968 afroecuatorianos, 56,45\% del total de 174.035 afrodescendientes en el país, lo que constituye el 7,2\% de todos los ecuatorianos. Además de que los afrodescendientes presentan un conjunto de indicadores sociales adversos que reflejan la desigualdad crítica y persistente, muestran una serie histórica de alta tasa de desempleo urbano. Así lo demuestran las encuestas de empleo del 2003 hasta el 2011, donde la tasa de desempleo urbano de la Población Económicamente Activa (PEA) afroecuatoriana es la más alta en todos los años, siendo superior en los jóvenes y las mujeres. De manera particular, según el 
Tabla 1. Esmeraldas y Ecuador: brechas en el ingreso laboral por horas

\begin{tabular}{lccc}
\hline REGIÓN & BRECHA EDU. PRIMARIA & BRECHA EDU. SECUNDARIA & BRECHA EDU. SUPERIOR \\
\hline Brecha Esmeraldas & 63,8 & 79,3 & 62,4 \\
Brecha país & 66,8 & 86,8 & 70,8 \\
\hline
\end{tabular}

Fuente: INEC (2006).

Censo de 2010, en el país los afroecuatorianos poseen una tasa de desempleo del 11\%, en tanto que en los indígenas llega al $4 \%$, en los mestizos al $8 \%$ y montubios $7 \%$, siendo el promedio de desempleo nacional de $7 \%$. Si se desglosa esta cifra por género, las mujeres afroecuatorianas muestran una tasa de desempleo de $17,5 \%$ y los hombres de $7 \%$; las mujeres ecuatorianas, por tanto, se encuentran desempleadas en una proporción de dos veces y medio mayor (INEC, 2011).

En Esmeraldas, la tasa de desempleo para el año 2006 era de 8,9\%, mientras que subempleo equivalía al 69,7\%. El desempleo de la provincia se concentra en las áreas de mayor densidad; es decir, las urbanas, con una clara brecha en detrimento de las mujeres. Tanto a nivel urbano como rural, el desempleo de la mujer casi duplica el de los hombres, lo que involucra problemas de exclusión, inequidad, desigualdad y falta de oportunidades en acceso al mercado laboral.

\section{EL CONTEXTO LABORAL EN ESMERALDAS}

El censo del 2010 determinó la categoría de ocupación de la PEA del cantón Esmeraldas de la siguiente manera: el $26 \%$ trabaja por cuenta propia y en la mayoría de los casos en el sector informal de la economía; el $20 \%$ es empleado privado; el 18\% es jornalero o peón; el 14\% es empleado del Estado o de los gobiernos locales; el 11\% no declara; el 4\% empleada doméstica; el $4 \%$ patrono, el $2 \%$ trabajo no remunerado y el $1 \%$ socio (INEC, 2010).

La participación de las mujeres en PEA remunerada decrece respecto de su participación en la PEA total. Ocurre en todos los casos con excepción del servicio doméstico, donde se mantiene igual. Esto es una muestra de la incidencia del trabajo no remunerado entre las mujeres. En el caso de los hombres sucede exactamente al revés: su participación en la PEA remunerada es mayor que en la PEA total. La mayor diferencia se presenta en el sector moderno, en el cual, si consideramos a todas las personas que trabajan, $56 \%$ son hombres, pero si nos referimos solamente a quienes son remuneradas, el porcentaje de hombres sube a $61,2 \%$ (INEC, 2006). Las brechas en el ingreso laboral mensual según grado de instrucción son muy reveladoras (ver Tabla 1).

Las mujeres con el mismo nivel de instrucción reciben en promedio entre el $62 \%$ y el $80 \%$ de lo que reciben los hombres por cada mes de trabajo (INEC, 2006). Las brechas en Esmeraldas son mayores que a nivel nacional, excepto en el caso de personas sin instrucción; la brecha en el nivel secundario es la menor, mientras que primaria y posgrado son casi iguales. A su vez, las mujeres dedican el triple de tiempo que los hombres a actividades relacionadas con la gestión del cuidado: en el aprovisionamiento, la preparación de alimentos, en el cuidado de hijas e hijos, cuidado de mayores, cuidado de la salud del grupo familiar y en la limpieza, entre otras. La situación de la provincia respecto al indicador es similar al promedio nacional, existe poca 
diferencia si comparamos las brechas de trabajo doméstico y trabajo voluntario, la brecha del cuidado de hijos o hijas sí es más elevada en Esmeraldas que en el país.

Lo reseñado constituye un panorama que contribuye a dificultar la autonomía de las mujeres: con la carga de trabajo de gestión del cuidado que soportan, sus posibilidades de participación en actividades laborales remuneradas se reducen, y las condiciones en las que las realizan son inequitativas respecto a los hombres. Además, el peso del trabajo reproductivo ocasiona dificultades para los ascensos y, en general, para los empleos a tiempo completo. Así, las mujeres esmeraldeñas dedican en promedio por semana $25 \mathrm{~h}$ y $21 \mathrm{~min}$ a actividades como cocinar, limpiar, lavar, cuidar niños y enfermos, mientras que los varones apenas dedican a las mismas actividades solamente $6 \mathrm{~h}$ y 2 min. Las mujeres trabajan dentro y fuera del hogar en promedio 77 horas a la semana, lo cual significa 20 h más que los hombres en las mismas condiciones. En el caso de las personas que no trabajan fuera del hogar, las mujeres trabajan 22 h más que los hombres.

Si bien los ingresos laborales masculinos pueden ser percibidos como familiares y las mujeres en muchos casos dependen completamente de ellos, en los barrios marginales de Esmeraldas, las mujeres tienen en una gran mayoría de casos la jefatura familiar y buscan variadas formas de obtener ingresos. Las mujeres que no disponen de ingresos propios ${ }^{10}$ son las que, con mayor probabilidad, tienen menos opciones de resistir la violencia ejercida contra ellas y las que más difícilmente pueden acceder a mayores niveles de educación y a tener maternidades optadas y saludables. En promedio, $68 \%$ de todas las mujeres esmeraldeñas mayores de 15 años carece de ingresos propios, frente a un $41 \%$ de hombres en la misma situación. Del total de personas sin ingresos propios en Esmeraldas, el $73 \%$ son mujeres. La tasa es marcadamente más alta entre la población entre 15 y 24 años porque se trata, en su mayoría, de personas que se encuentran todavía estudiando. Pero entre las mujeres mayores de 60 años la tasa es muy parecida, lo que refleja su dependencia en otras personas durante una etapa vulnerable de la existencia.

Los mayores frenos para la igualdad y la autonomía de las mujeres de Esmeraldas se encuentran en el ámbito económico. No solo que es difícil ingresar al mercado laboral, sino que mientras mayor educación alcanzan las mujeres, aumenta la brecha de ingresos respecto de los hombres. Aun si tienen la misma instrucción, edad y experiencia, las mujeres ganan menos que los hombres. Si bien para toda persona un incremento en el nivel educativo se refleja en un incremento en los ingresos laborales, ese incremento es aún mayor para los hombres que para las mujeres. Además, las mujeres experimentan una situación de sobrecarga de trabajo, dedicando el triple de tiempo que los hombres a la gestión del cuidado, que si bien se invisibiliza económicamente, es precisamente lo que permite la reproducción social; es decir, «la producción de todas las condiciones de producción», (Turner y Brownhill, 2006).

\section{LOS BARRIOS DEL SUR Y CENTRO DE ESMERALDAS}

Con base a la investigación cualitativa realizada en Esmeraldas en 2014-2015 se constató que en la gran mayoría de hogares en los barrios del sur aledaños a la refinería, y también en ciertos barrios del centro, las mujeres ejercen la jefatura familiar. Esto corrobora y sobrepasa el dato 
de la jefatura femenina en el cantón Esmeraldas, que alcanza el 57,8 \% según el último censo nacional. Se observa que las mujeres son las que permanecen al frente de sus unidades domésticas, mientras los hombres tienen una presencia más itinerante. Las mujeres afrodescendientes pasan por distintas uniones y compromisos a lo largo de sus vidas y son quienes se ocupan del cuidado de los hijos, del aprovisionamiento de agua (y otros recursos) y también de la generación de ingresos económicos. Son invisibilizadas en el PEA, pero ejercen cotidianamente el trabajo reproductivo necesario y en el sector informal de la economía.

En entrevistas a mujeres lideresas e integrantes de directivas barriales, la percepción es que la situación de la mujer en los barrios es especialmente difícil, puesto que son las que sienten los mayores impactos del desempleo. Consideran que se efectúa un nulo reconocimiento del trabajo en el hogar y existe una sobrecarga no solo del trabajo en el hogar, sino también del trabajo comunitario, ya que la mayoría de los dirigentes en estos barrios son mujeres. Igualmente son las mujeres quienes perciben de manera más intensa el impacto ambiental de la refinería y de otras actividades - como la maderera con la empresa de Contrachapados de Esmeraldas s. A. (CODESA) - , con efectos de manera especial en niños y niñas, a quienes deben atender y cuidar. Los barrios del sur de Esmeraldas comprenden dos partes identificadas por la población:

Toda el área de los barrios Unidos Somos Más, 15 de marzo, Lucha de los Pobres, los barrios de La Propicia, la urbanización La Concordia, las villas de Petroecuador y el gran valle de San Rafael - barrio que empezó como un gran barrio que se subdividió en muchos barrios, ahora son algo más de 50 barrios que si se suman a la zona mencionada inicialmente, superan los 70 barrios del sur de la ciudad-. Esta zona es de establecimiento relativamente reciente y se fue conformando gracias a varios factores, mayormente la poca disponibilidad de espacios constructivos en el centro de Esmeraldas. Las extensas tierras del sur de Esmeraldas también estaban en manos de pocos propietarios; eran en su mayoría haciendas agrícolas que sufrieron invasiones desde la década del 7o. Tales aspectos impulsaron distintos flujos migratorios que condicionaron la evolución de cada uno de los barrios, algunos con servicios básicos (aunque no siempre de calidad) y otros creciendo en desorden y con mucha precariedad. Esta última circunstancia motivó el surgimiento de organizaciones sociales en un número superior a otros sectores de la ciudad, cuyos objetivos eran principalmente luchar por la dotación de servicios básicos y por la propiedad de la tierra.

En el área contigua a los barrios del sur se encuentran las principales infraestructuras industriales de la ciudad: la Refinería Estatal y TermoEsmeraldas. Cuando se construyó la primera - en 1974 por el consorcio Sumitomo Chiyoda-, no existían habitantes en esa zona. En un principio, solo unos pocos trabajadores locales que desempeñaban las tareas más duras de construcción de la refinería se fueron instalando en las cercanías. Para 1977, cuando la refinería entra en funcionamiento, varias familias ya estaban ubicadas en los alrededores, siendo los más antiguos los barrios de La Propicia 1, que se conformó en terrenos que eran propiedad de Delfina Torres de Concha, y el barrio Unidos Somos Más, conocido como codesa debido a la fábrica de contrachapados existente en el sector. Cuando TermoEsmeraldas empezó a construirse en 1982, ya la zona presentaba un rápido crecimiento demográfico. Otro afluente migratorio se dio a raíz del fenómeno del Niño de 1998, que produjo deslizamientos en el centro de la ciudad en barrios como el 13 de abril, el Gatazo y el Tercer Piso, obligando a cientos de 
familias a reubicarse en la zona sur de la ciudad. El sur se fue configurando en condiciones de precariedad en el acceso a servicios básicos, mala calidad de las vías, inseguridad, paisaje urbano desorganizado, ausencia de unidades de salud completas y, por otra parte, su población directamente expuesta a la contaminación de aire y suelos, en gran medida por la presencia de la refinería y TermoEsmeraldas, pero también por la incidencia de la procesadora de aceite, por CODEsA y el terminal terrestre.

La población de los barrios del sur en su gran mayoría está compuesta por trabajadores formales e informales, mientras que una minoría son servidores públicos. Esta población de trabajadores se emplea en trabajos ocasionales; las mujeres principalmente laboran en el sector de servicios, en la venta de alimentos preparados y como lavanderas, empleadas domésticas, costureras, comercio menor, entre otros. Algunas mujeres han conformado microempresas y han instalado pequeños negocios en sus hogares. Existe también un alto porcentaje de desempleo que se vincula con el incremento de la violencia y por lo tanto de la inseguridad, igualmente con el aumento del alcoholismo, la drogadicción y maternidad precoz. En estos lugares, y en particular donde vive la población de menos recursos, se organizan los barrios en comités promejoras que impulsan y fomentar mejoras en sus sectores: acceso integral a los servicios básicos, garantizar el goce del derecho a la salud, a un ambiente saludable, mayor seguridad. Para algunos de los barrios la preocupación fundamental de las directivas es lograr la propiedad de sus tierras, puesto que sin ésta, no pueden acceder a beneficios ni créditos en el sistema financiero.

En el caso del centro de la ciudad se realizó la investigación en el barrio del Kilombo, donde destaca el liderazgo femenino tanto en el barrio como en sus hogares. Este barrio se conformó con mujeres que salieron de barrios bajos a partir del fenómeno del Niño y por la violencia que experimentaban para conseguir una vida mejor para sus hijos y familia. La historia del barrio y de sus fundadoras es la historia de la lucha por los servicios públicos, y aunque aparece alguna conexión con el circuito petrolero al acceder a alcantarillado y agua ofrecidos por el municipio con recursos del ocp, la dotación no obstante es indirecta. Es más, a pesar de contar con tubería, no hay agua y los residentes de Kilombo se tienen que abastecerse como varios otros barrios, con agua de tanqueros.

En el barrio viven las mujeres conocidas como «morocheras», quienes han emprendido un negocio que consiste en preparar y vender morocho - bebida dulce de maíz y leche- y empanadas fritas de harina con queso. Las morocheras suelen situarse cerca de los hospitales y centros de salud, que son en el centro norte de la ciudad. La inversión en materia prima para un día de trabajo va de 20 a 50 dólares, y la utilidad es el doble de lo invertido. Las ventas de morocho suelen ser bastante buenas y en el negocio participa toda la familia en la preparación del producto. Además, en los puestos de ventas, las mujeres no están solas, sino que las acompañan los hijos mayores, las hijas, los niños y en uno que otro caso los maridos. En general, la venta de morocho representa la única fuente de ingresos en el hogar, lo que da una visibilidad especial a la jefatura femenina de los hogares.

Respecto a las fuentes de trabajo creadas por las actividades de refinación en la ciudad, la empresa estatal siempre ha trabajado con empresas de servicios de fuera de la provincia; es decir, pocas utilidades - públicas o privadas - de las generadas al pertenecer al circuito 
petrolero se quedaron en la ciudad tras treinta y ocho años de la inauguración de la refinería. La mayoría de trabajadores locales que han logrado entrar a la empresa estatal petrolera son hombres; las mujeres han participado como parte de las empresas prestadoras de servicios, o sea, marginalmente. Las mujeres del barrio Kilombo no tienen expectativas respecto a que la situación económica mejore y tener más oportunidades de empleo. El sector petrolero no les representa esperanza de progreso. Frente a esto, implementan una estrategia cultural, el «vivir el hoy» es una práctica casi obligada: hay que disfrutar el momento. Esta actitud las mueve frente a una realidad caótica y discriminadora en las que, además, se encuentran con la responsabilidad adicional de cuidar a la salud de sus familiares amenazada por la contaminación ambiental que produce la refinería. Está claro que la situación de las mujeres en Esmeraldas no se mejoró por la integración de su ciudad al circuito petrolero, sino que se precarizó aún más, cargándoles con trabajos marginados e informales y labores adicionales de cuidado.

\section{MUJERES KICHWAS Y MESTIZAS EN LAS CIUDADES DEL MILENIO}

(EN EL NORORIENTE AMAZÓNICO)

En lugares de la Amazonía más recientemente integrados a la industria petrolera el gobierno de Alianza País ha publicitado la inversión y construcción de obras vinculadas a su política de inclusión social en zonas estratégicas para la exploración y explotación de recursos naturales no renovables. La denominada «Nueva Amazonía» se construiría con recursos de la renta petrolera y minera para sacar de una histórica exclusión de las poblaciones amazónicas. Al 2014 se habría invertido un total de USD 632,4 millones de dólares en 846 proyectos de desarrollo integral en las 6 provincias de la Amazonía; con una concentración mayor en las provincias de Sucumbíos y Orellana. ${ }^{11}$ Entre las obras emblemáticas se encuentran las Ciudades del Milenio (Informe de Rendición de Cuentas de EE, 2014).

En este acápite haremos mención a dos comunidades, Playas de Cuyabeno y Pañacocha, que se transformaron en Ciudades del Milenio en la provincia de Sucumbíos, en la Amazonía norte. La primera se localiza en la confluencia entre los ríos Aguarico y Cuyabeno y fue inaugurada como Ciudad del Milenio el 1. ${ }^{\circ}$ de octubre de 2013; la segunda es un importante puerto a lo largo de la ribera del Napo por su situación en el eje fluvial Coca-Nuevo Rocafuerte y fue inaugurada como Cuidad del Milenio a principios del 2014. Andes del 2012, ambas eran comunidades predominantemente kichwas, no obstante, también conformadas por población mestiza. ${ }^{12}$ La construcción de las Ciudades del Milenio es el resultado de un proceso de negociación entre las dirigencias y las comunidades de Playas de Cuyabeno, Pañacocha y Pucapeña, insertas dentro del bloque 12 (Edén-Yuturi), que a la salida de Occidental (oxy), pasó a ser operado por Petroamazonas desde el 2007.

Cuando PetroAmazonas ingresó en el 2008 a Playas de Cuyabeno para socializar el proyecto extractivo, hubo resistencia principalmente por las negociaciones directas de la empresa con un grupo de familias de Santa Elena ubicada en el territorio de Playas, saltándose instancias organizativas. Intervinieron en las negociaciones para el inicio de actividades extractivas, el gobierno a través de la Secretaría Nacional de Planificación (sEnplades) y la Vicepresidencia de la República. Las comunidades tenían una serie de demandas para acceder a compensaciones 
y planteaban no querer que se repitan las viejas políticas de relacionamiento empresas-comunidades que se plasmaban en pequeños proyectos o entrega de ciertos materiales para escuelas o viviendas. Buscaban una compensación más equitativa, acceso a servicios básicos, mejoramiento de las condiciones de la infraestructura educativa hasta bachillerato y la generación de fuentes de empleo. ${ }^{13}$ Se convino con la dirigencia de estas comunidades la construcción de las Ciudades del Milenio y el acuerdo que se selló en 2010 mediante una ceremonia en la que se entregó a ambas comunidades un cheque simbólico por USD 22 millones.

En el 2012 Petroamazonas comenzó a operar el campo Pañacocha, y a la par que se realizaba la socialización de la construcción de las Ciudades del Milenio en ambas comunidades, se perforó el pozo Yana Urku en Pañacocha y se firmó un convenio con Petroamazonas para que la línea de flujo, que atraviesa el territorio de la Comuna Kichwa Pañacocha, llegue al Aguarico además de la realización de un nuevo estudio sísmico para determinar la presencia de pozos positivos en el territorio de la Comuna. Las ciudades con dotación de viviendas para las familias comenzaron a ser habitadas en el 2013. Cuentan con energía eléctrica, agua potable, acceso a internet y telefonía, computadores, cocinas de inducción, refrigerador; las ciudades están dotadas adicionalmente de una Unidad Educativa del Milenio igualmente equipadas con tecnología, laboratorios, biblioteca. La parafernalia arquitectónica y de servicios materializa, a decir del gobierno, la inclusión social ofrecida, y el modelo podría replicarse en otras zonas más de la Amazonía donde tienen lugar actividades extractivas — está actualmente en construcción una tercera Ciudad del Milenio en la comunidad A’i Cofán de Dureno.

Además de la serie de transformaciones en el paisaje y ecosistemas - como el drenaje y relleno de áreas de moretales en los antes pequeños centros poblados de las comunidades- se han dado una serie cambios en la cotidianidad de las familias con contrastes fuertes entre generaciones: los adultos mayores prefieren permanecer en las casas de las fincas en el territorio comunitario, mientras los jóvenes prefieren pasar en las urbanizaciones nuevas. En lo que sigue, nos concentraremos en analizar las dinámicas que se han generado en torno al trabajo, en las relaciones de género y en la relación reproducción-producción con atención a la situación de las mujeres en las nuevas Ciudades del Milenio.

\section{LA ATRACCIÓN HACIA FUENTES DE EMPLEO EN LA INDUSTRIA EXTRACTIVA: UNA ILUSIÓN PASAJERA}

En general, antes del 2013 las familias kichwas y mestizas de Pañacocha y Playas de Cuyabeno se dedicaban a actividades vinculadas al manejo de fincas, con una economía de subsistencia basada en agricultura con producción de yuca, plátano, maíz que combinaba un patrón de subsistencia familiar con venta para el mercado. Las familias de Playas de Cuyabeno vendían principalmente maíz en Tarapoa, Rocafuerte y Lago Agrio. Algunas familias criaban también gallinas y pollos y complementaban su provisión alimentaria con cacería y pesca. La mayor producción se destinaba al consumo familiar y otra parte a su comercialización. En ambas comunidades, las mujeres siempre han sido las que han sustentado el aprovisionamiento familiar, ya que algunos hombres se ausentaban de la comunidad por temporadas para realizar trabajos al jornal en agricultura o trabajos en empresas petroleras en otros lugares de Sucumbíos. El proceso 
Tabla 2. Rama de actividad por etnia (Parroquia Cuyabeno)

\begin{tabular}{lcccc}
\hline & \multicolumn{2}{c}{ HOMBRE } & \multicolumn{2}{c}{ MUJER } \\
\hline ACTIVIDAD & INDÍGENA & MESTIZO/A & INDÍGENA & MESTIzo/A \\
\hline Agricultura, ganadería, & 26,1 & 25 & 17,2 & 8,3 \\
silvicultura y pesca & & & & \\
Enseñanza & 3,0 & 8,3 & 0,0 & 8,3 \\
Construcción & 0,0 & 8,3 & 0,0 & 0,0 \\
Transporte y almacenamiento & 1,5 & 0,0 & 0,0 & 0,0 \\
Actividades de alojamiento y & 0,0 & 0,0 & 0,0 & 16,7 \\
servicio de comidas & & & & \\
\hline
\end{tabular}

Fuente: Geovanny Castro (Proyecto Fda. Ecologías Políticas de la Diferencia) con base a INEC (2010).

muestra cómo la producción capitalista atada al extractivismo en su ampliación y expansión irrumpe y subordina a formas económicas de reproducción simple (Schmink y Wood, 1987).

En Playas de Cuyabeno, las familias también se insertaron desde décadas previas en actividades de turismo; cuando funcionaba el Flotel Orellana, los comunitarios proporcionaban servicios de guías, canoeros y remeros, y las mujeres se dedicaban a elaborar artesanías para la venta a los turistas. En el caso de Pañacocha, en tiempos anteriores a la construcción de la Ciudad del Milenio, las familias vivían en fincas de 50 hectáreas cada una localizada a lo largo de la ribera del Napo. El cambio a un patrón urbanizado con la construcción de la ciudad, puso en juego una serie de estrategias en ambos lugares. Uno de los efectos fue la disminución de las prácticas agrícolas, por la vinculación de los comuneros, hombres en su mayoría, al trabajo en las empresas. Los comuneros entrevistados expresaron su preferencia por tener un empleo remunerado en las empresas que dedicarse a la agricultura, debido a que ésta supone un ingreso variable. Esto generó el desplazamiento de la agricultura a una actividad secundaria. En este encuadre, las mujeres se tornaron dependientes de los ingresos de sus cónyuges para la provisión de alimentos para sus grupos familiares. Esto, en la medida en que las Ciudades del Milenio tenían protocolos que no permitían a las mujeres criar animales ni tener cultivos contiguos a las viviendas. Así mismo, la nueva tecnología en sus casas no les permite preparar alimentos de forma tradicional (carne de monte asada, maitos), ni elaborar la chicha.

A continuación se presentan algunas estadísticas con base a los datos del Censo INEC 2010 que muestran la configuración de la PEA por rama de actividad en las parroquias de Cuyabeno y de Pañacocha y la autoadscripción indígena o mestiza. A nivel de parroquia, Cuyabeno cuenta con un $87,8 \%$ de población indígena, $11,5 \%$ de mestiza. De la población autoadscrita indígena, $62 \%$ es kichwa. De la población general en la parroquia de Cuyabeno, 48,5\% es hombre indígena, $39,3 \%$ es mujer indígena, $6,6 \%$ es hombre mestizo y 4,8\% mujer mestiza (ver Tabla 2 ).

Conforme al cuadro con datos de la parroquia Cuyabeno se puede observar que los hombres indígenas (en 26,1\%) y mestizos (en 25\%) tienen como principal rama de actividad económica la agricultura combinada con ganadería, silvicultura y pesca. En un rango menor constan actividades como enseñanza y transporte. En el caso de las mujeres indígenas, su rama de actividad es decididamente la agricultura, silvicultura y pesca (17,2\%), mientras las mujeres 
mestizas tienen una dedicación a actividades de servicio de comidas y alojamiento (16,7\%) seguido de agricultura, ganadería y pesca y de enseñanza.

En cuanto a las categorías de ocupación (ver Tabla 3), los hombres indígenas en un 37,9\% realizan actividades económicas (agrícola, pecuaria, silvicultura) por cuenta propia, lo mismo en el caso de las mujeres indígenas en un 30,3\%. Los hombres mestizos en un $25 \%$ y mujeres mestizas trabajan por cuenta propia en igual porcentaje. Los hombres indígenas trabajan como empleados del sector público en un 10,6\%, como empleado obrero y privado en un 4,5\% y como jornaleros en $2,3 \%$. Las mujeres indígenas en $3 \%$ trabajan como empleadas de sector público. En contraste, los hombres mestizos en $25 \%$ trabajan como empleado de sector público, como obreros privados y como jornaleros en porcentaje un tanto mayor a los hombres indígenas, en $8,3 \% .16,7 \%$ de las mujeres mestizas trabajan como empleadas del sector público (INEC, 2010). Mientras que la tasa de participación laboral global ${ }^{14}$ en el caso de hombres indígenas es de $55,1 \%$, mujeres indígenas de $45,2 \%$, hombres mestizos en $40 \%$ y mujeres mestizas en $35,7 \%$ (INEC, 2010).

En lo que respecta a Pañacocha, el 77,8\% de la población se autoidentifica como indígena, mientras $19,1 \%$ se identifica como mestiza. El 40,8\% son mujeres indígenas, 36,9\% es hombre indígena; $6,9 \%$ son mujeres mestizas, $12,2 \%$ son hombres mestizos. En cuanto a rama de actividad, el 29,2\% de hombres indígenas se dedican a la agricultura, ganadería, silvicultura y pesca; 22,4\% de las mujeres indígenas se dedican a esta misma actividad. El 10,2\% de hombres mestizos se dedican a esta actividad (ver Tabla 4 ).

Conforme a la categoría de ocupación por etnias, el 29,2\% de hombres indígenas trabajan por cuenta propia, como jornalero 3,2\%; mientras que el 20,3\% de mujeres indígenas trabajan por cuenta propia, o,3\% como jornalera/peón. En el caso de hombre mestizos, trabajan por cuenta propia 9,8\%, 1,9\% como peón al jornal; las mujeres mestizas por cuenta propia se registra $0,3 \%$ (ver Tabla 5). En cuanto a tasa de participación laboral global, 69,9\% corresponde a hombres indígenas, $46,1 \%$ a mujeres indígenas; $77,6 \%$ a hombres mestizos y $14,7 \%$ a mujeres mestizas (INEC, 2010).

Siguiendo con la argumentación previa, la generación de fuentes de empleo en la industria extractiva fue un interés de los pobladores en ambas comunidades, lo que llevó a que no se dimensionen los efectos en el territorio, ni los posibles impactos ambientales. En el caso de Playas de Cuyabeno, desde el 2008, cuando empezaron las visitas de parte de Petroamazonas, se generó la expectativa del inicio de actividades petroleras y se produjo un repoblamiento de la comunidad, ya que algunos hijos de las familias vivían para entonces en Lago Agrio y otras ciudades amazónicas.

En el caso de Pañacocha ha sido un territorio con una larga data de intervención sísmica, desde la década de los setenta hasta el último estudio realizado en 2010. Varias empresas extranjeras han pasado por Pañacocha y por Edén. Solamente la empresa china BGP gestionó programas de indemnizaciones y compensaciones por los posibles impactos ambientales causados durante la realización de los estudios de sísmica. Tras la salida de los misioneros capuchinos en los setenta, la población se vinculó a las empresas extranjeras como mano de obra no calificada en la apertura de trochas, construcción, limpieza, entre otras actividades. Por tanto, los pobladores de Pañacocha, principalmente los hombres de la comunidad, ya habían tenido cierta relación de dependencia hacia las empresas petroleras en términos laborales antes del 
Tabla 3. Categoría de ocupación (Parroquia Cuyabeno)

\begin{tabular}{lcccc}
\hline & \multicolumn{2}{c}{ HOMBRE } & \multicolumn{2}{c}{ MUJER } \\
\hline CATEGoría & INDÍGENA & MESTIZO/A & INDÍGENA & MESTIZO/A \\
\hline Cuenta propia & 37,9 & 25,0 & 30,3 & 25,0 \\
Empleado/a del sector públic & 10,0 & 8,3 & 3,0 & 16,7 \\
Empleado/a privado & 4,5 & 8,3 & 0,0 & 0,0 \\
Jornalero/a o peón & 2,3 & 8,3 & 0,0 & 0,0 \\
\hline
\end{tabular}

Fuente: Geovanny Castro (Proyecto Fda. Ecologías Políticas de la Diferencia) con base a INEC (2010).

Tabla 4. Rama de actividad económica por etnia (Parroquia Pañacocha)

\begin{tabular}{lcccc}
\hline & \multicolumn{2}{c}{ HOMBRE } & \multicolumn{2}{c}{ MUJER } \\
\hline ACTIVIDAD & INDÍGENA & MESTIZO/A & INDÍGENA & MESTIZO/A \\
\hline $\begin{array}{l}\text { Agricultura, ganadería, silvicul- } \\
\text { tura y pesca }\end{array}$ & 29,2 & 10,2 & 22,4 & 0,0 \\
No declarado & & & & 0,6 \\
\hline
\end{tabular}

Fuente: Geovanny Castro (Proyecto Fda. Ecologías Políticas de la Diferencia) con base a INEC (2010).

Tabla 5. Rama de actividad económica por etnia (Parroquia Pañacocha)

\begin{tabular}{lcccc}
\hline & \multicolumn{2}{c}{ HOMBRE } & \multicolumn{2}{c}{ MUJER } \\
\hline CATEGORÍA & INDÍGENA & MESTIZO/A & INDÍGENA & MESTIZo/A \\
\hline Cuenta propia & 29,2 & 9,8 & 20,3 & 0,3 \\
Se ignora & 7,3 & 1,3 & 9,8 & 0,6 \\
Jornalero/a o peón & 3,2 & 1,9 & 0,3 & 0,0 \\
\hline
\end{tabular}

Fuente: Geovanny Castro (Proyecto Fda. Ecologías Políticas de la Diferencia) con base a INEC (2010).

inicio de actividades extractivas en su territorio. Además, Pañacocha comparte vecindad con el campo Edén-Yuturi - el campo de mayor producción en el nororiente ecuatoriano- operado por la Occidental desde la década de los noventa hasta el 2006, a la que también se vincularon los pobladores de Pañacocha como mano de obra.

Con la reciente implementación de actividades de exploración sísmica y la instalación de plataformas de extracción, se generaron puestos de empleo temporal para la población masculina tanto en Playas de Cuyabeno como en Pañacocha, como mano de obra no calificada y en actividades de transporte fluvial. Esto en la medida en que, con las compensaciones recibidas por permitir actividades de exploración sísmica, algunas familias adquirieron canoas y motores fuera de borda, en otros casos lanchas modernas y deslizadores para prestar servicios de transporte a operarios de la empresa Petroamazonas y a empresas contratistas. En el caso de los habitantes de Santa Elena, dentro del territorio de Playas pero más cercano a la extracción petrolera, la familia Vásquez compró lanchas y vehículos para transportar operarios por los caminos que conducen a los pozos. En Playas de Cuyabeno, las mujeres participaron poco en estas actividades. A diferencia de 
Pañacocha en donde tanto hombres como mujeres se insertaron en actividades de construcción de la Ciudad del Milenio. Por lo tanto, las mujeres de Playas se sentían más dependientes del ingreso de sus cónyuges y limitadas en su autonomía económica. Algunas habitantes de Playas instalaron comedores para proporcionar servicios a la gama de actores que se insertaron temporalmente en el territorio - operarios de Petroamazonas, empresas de servicios y personal de la empresa constructora CVA-. En el caso de Pañacocha, en cambio, las mujeres se insertaron en actividades de limpieza, lavado de ropa y el arreglo de habitaciones en el hotel ${ }^{15}$ que cobró dinamismo y se mantuvo en actividad durante la construcción de la Ciudad del Milenio, recibiendo a operarios de cva16.

Fue especialmente aguda la experiencia de las mujeres de Playas de Cuyabeno quienes vieron reducirse sus posibilidades de aprovisionamiento autónomo. Las jóvenes madres, con niños de escuela, se tornaron cada vez más dependientes de los hombres insertados en la economía monetaria. Si antes las mujeres cuidaban animales y huertos cerca a sus casas y en sus fincas, su ocupación de las casas en la Ciudad del Milenio y la compra de comida procesada en tiendas significó para muchas de ellas el abandono de la chacra y una menor participación en la provisión de la alimentación familiar.

Tanto en Playas como en Pañacocha, las mujeres en sus nuevos espacios urbanos sintieron que sobre ellas descansaban expectativas en lo que se refiere al mantenimiento de la urbanidad que ahora debía permanecer adecuado a ciertos protocolos. Las Ciudades del Milenio interrumpieron en la relación de las mujeres con las fincas y la selva; como se ha mencionado, principalmente las jóvenes tuvieron que permanecer más en sus casas para estar pendientes de sus hijos de edad escolar. Otras tuvieron que transitar entre sus casas en las Ciudades del Milenio y sus casas de finca. Quienes se alejaron más de la actividad del cultivo de chacras y crianza de animales vieron disminuida su autonomía alimentaria, con una mayor dependencia de productos procesados. Esto se muestra como efecto de la desposesión. Se produjeron, asimismo, un aumento en las diferenciaciones económicas y generacionales al interior de la comunidad, basado en el acceso a dinero y consumo.

Las mujeres - sus hijas y sus hijos en la Ciudad del Milenio- han experimentado fracturas en los vínculos y la interdependencia con la naturaleza, principalmente los más jóvenes, que dejaron de participar de actividades agrícolas, de pesca y cacería, distanciándose de las fincas y de transitar por la selva. En las actividades cotidianas se ha producido cierto alejamiento en la transmisión de saberes vinculados a las prácticas de manejo, lo que no sólo nos habla de la pérdida del acceso a modos de producción y reproducción fundados sobre recursos vitales, sino que implica transformaciones en las subjetividades sociales acordes con la continuidad del sistema capitalista. A lo que asistimos en la Amazonía ecuatoriana es a mutaciones en las relaciones articuladas entre la producción y la reproducción, y en las relaciones humano-naturaleza. El confinamiento del trabajo de las mujeres a la reproducción social domesticada - a la esfera de sus nuevas viviendas en las Ciudades del Milenio- no solo ha afectado a su autonomía y poder social y económico, sino que también contrajo su participación en los ciclos de la regeneración de la vida social y de la naturaleza. El modelo urbanizado replica lo que Federici (2004) menciona que ocurre con la acumulación original del capital: ha derivado en el mayor encierro de las mujeres en el espacio doméstico, haciéndoles perder 
control con respecto a sus vidas, forzando una ruptura respecto a la interdependencia y participación que tenían en el sistema socioeconómico basado en actividades dirigidas a la reproducción y el sostenimiento de la vida.

\section{PASÓ LA EUFORIA: AUSENCIA DE FUENTES DE EMPLEO REMUNERADO (NO CALIFICADO) EN LA INDUSTRIA PETROLERA}

En el 2014 había alrededor de diez socios de la comunidad de Playas de Cuyabeno que tenían trabajos específicos como guardianes o jardineros en la Ciudad del Milenio, o quienes eran ayudantes de operadores y motoristas. Solo un año después, en 2015, no llegan a más de tres las personas de Playas vinculadas contractualmente con Petroamazonas. Esto significa que la presencia de la compañía petrolera no ha significado una oferta estable de plazas de trabajo como fue el requerimiento de la población a la empresa cuando se iniciaron las exploraciones en el 2010. Una vez instaladas las plataformas y que continuaron las actividades de extracción de petróleo, la empresa dejó de contratar servicios y trabajadores de las comunidades. La expectativa de generación de fuentes de empleo se vería frustrada en el 2015. Algunas familias para complementar ingresos monetarios implementaron en la parte baja de sus casas del milenio, bazares, tiendas y cantinas. Esto también generó que nuevamente las familias en Playas de Cuyabeno y Pañacocha se tengan que volcar a su trabajo y actividades en las fincas.

Lo más complejo a todo este proceso es que las familias de ambas Ciudades del Milenio tendrán que solventar los gastos correspondientes al pago de servicios a los que han accedido. Si bien en primera instancia la empresa PetroAmazonas cubría gastos de tratamiento de agua, jardines, manejo de la basura, esto posteriormente tendrán que ser solventados por los gobiernos locales (juntas parroquiales), y el consumo de energía, agua potable, acceso a internet y telefonía cubierto por cada familia. Al no tener una fuente de empleo remunerado estable, la situación de las familias es frágil, en el sentido de que adquirieron nuevas necesidades, se integraron al consumo de ciertos servicios, pero no están plenamente integrados al mercado laboral, ni cuentan con ingresos económicos que les permita solventar lo accedido.

Las Ciudades del Milenio representan las experiencias de una inclusión al modelo de desarollo homogeneizador, que lleva a nuevas dependencias materiales y subjetivas que precarizan a las poblaciones ante la volatilidad de los precios del petróleo en el mercado global y la supeditación al modelo primario-exportador. Las mujeres en este escenario no han sido integradas, sino a través de trabajos eventuales - lavado de ropa, preparación de alimentos para personeros de PetroAmazonas, la constructora CvA u otros operarios que han transitado por sus territorios-, y de ellas ha dependido el aprovisionamiento familiar, principalmente de aquellas que han permanecido más articuladas a sus fincas. Una vez que la euforia de las compensaciones y la incipiente oferta laboral se han desdibujado, las familias han tenido que volcarse nuevamente al trabajo en la producción de sus fincas. Las mujeres, principalmente las adultas y mayores, dan el soporte ante la pendulante economía local con su trabajo de cultivo en chacra y con el cuidado de animales, asegurando el sustento de sus familias.

Dadas las dinámicas de trabajo y economía local que implican, se evidencia que los proyectos de desarrollo de las Ciudades del Milenio encubren formas de despojo del territorio y de 
la relación sociedad-naturaleza, bajo la aparente compensación e inclusión social de las poblaciones. La desposesión territorial hace parte de los mecanismos de la acumulación del capital, en sus continuas reediciones en el sur global. Éstas no se pueden entender solamente como parte de reconfiguraciones sociales, políticas y económicas. Federici (2004) demuestra las maneras en que la instauración del capitalismo depende de la escisión entre trabajo productivo y reproductivo, así como del despojo del poder de la mujer tras la forzada expulsión de los campesinos de sus tierras; para el caso abordado, de la desposesión de territorios étnicos.

\section{CONCLUSIONES}

En el caso amazónico, el modelo petrolero genera reordenamientos territoriales basados en el control de recursos y poblaciones; irrumpiendo en dinámicas territoriales locales y de comunidades indígenas, en sus propias formas de gestión del territorio en las que las mujeres han tenido un papel activo y autónomo en la provisión de recursos para la subsistencia.

En el caso de Esmeraldas, a pesar de la presencia por varias décadas de actividades de refinamiento de petróleo, los barrios de la ciudad cercanos a la refinería han permanecido relegados del acceso a fuentes de empleo vinculados a la actividad petrolera y excluidos de la participación en la planificación de las políticas de inclusión social, mientras continúa su exposición a la contaminación y sufrimiento ambiental. Así mismo, existen muy limitadas fuentes de empleo para la población relacionada con la industria del refinamiento petrolero; esto es más notorio en el caso de las mujeres, relegadas por falta de calificación técnica a trabajos informales y a subempleo en la ciudad. En un contexto de carencia en la provisión de servicios básicos, a pesar de los ingentes ingresos petroleros, las mujeres ensayan estrategias disímiles para el sustento de sus grupos familiares, de los cuales generalmente ejercen jefatura.

En el nororiente de la Amazonía en la provincia de Sucumbíos, la transformación de comunidades en Ciudades del Milenio significó una acelerada incorporación de la población a la economía monetaria a través del estímulo del consumo de servicios, energía, tecnología, provocando transformaciones en las relaciones de género y rupturas intergeneracionales. Se han transformado los roles económico-productivos y reproductivos en la comunidad, las relaciones con el territorio y la naturaleza y las formas de sostenimiento de las familias. Sobre todo, ha significado para las mujeres jóvenes principalmente perder la autonomía que antes tenían en el manejo de agroecosistemas en sus chacras y fincas, así como por la crianza de animales destinados a la subsistencia y en menor medida a la comercialización. En este sentido, su dependencia hacia el trabajo remunerado de los hombres se ha incrementado, ya que la mano de obra masculina se empleó en la instalación de las actividades extractivas en servicios terciarios para PetroAmazonas; trabajos que, sin embargo, no se sostienen en el tiempo. En tiempos de crisis económica nacional y de la caída en los precios de los commodities, esto podría significar una oportunidad para las mujeres al fortalecer su trabajo reproductivo, en pro de la fertilidad y los comunes (Turner y Brownhill, 2006, p. 89). Tanto en los lugares de la Amazonía y de la Costa ecuatoriana que se encuentran integrados al circuito petrolero, las mujeres indígenas kichwas, mestizas y afrodescendientes siguen siendo el soporte del trabajo reproductivo, 
de la gestión del cuidado y de formas de aprovisionamiento. El capital no obstante no recompensa el costo de la reproducción.

\section{NOTAS}

1 El artículo ha sido elaborado con insumos de dos años de investigación (2014-2015) en el marco del Proyecto Ecologías Políticas de la Diferencia en lugares del circuito petrolero en Costa y Amazonía efectuado desde flacso, sede Ecuador. En la investigación de campo en los sitios en mención participaron Angus Lyall, Nancy Carrión (Playas de Cuyabeno), Natalia Valdivieso (Pañacocha), Piedad Ortiz y Eduardo Morcillo (Esmeraldas).

2 En el siglo xx hubo varias crisis del petróleo derivadas del problema de la excesiva dependencia de un producto de exportación: en 1983 en un período de política económica neoliberal, coadyuvó a nivel local el Fenómeno del Niño, y a nivel latinoamericano la crisis de la deuda. En 1987 se produjo una nueva caída del precio del petróleo, atado a la crisis de deuda externa. La crisis económica nacional fue coadyuvada por el terremoto y rotura del oleoducto. En 1999 se presentó otra caída precio del petróleo, lo que se dio en un período de política económico neoliberal recesiva, a nivel internacional crisis mexicano, asiática y rusa, período en que hubo crisis de la deuda externa, Ecuador entró en moratoria y corrupción, a lo que se coligó el Fenómeno del Niño.

3 El aumento del volumen de exportaciones de commodities, según Lander, se ha dado en los últimos años también en países como Bolivia y Venezuela. En Ecuador, la participación de los productos primarios en proporción del valor total de las exportaciones «varió entre 2005 con 90,4\% al 2010 con 90,2\%» (Lander, 2013, p. 14).

4 Este cambio se ha visto más en la inversión destinada a la implementación de grandes proyectos hidroeléctricos, con los cuales el país disminuiría su dependencia de recursos fósiles para la generación de energía, y potenciaría la venta de energía a países vecinos. El apoyo gubernamental a la diversificación económica, sin embargo, no se ha visto materializado.

5 Parte de dicha profundización refleja la decisión en agosto del 2013, tomada por el presidente Rafael Correa, de explotar el campo petrolero Ishpingo-Tambococha-Tiputini (ITT) en la Reserva de Biósfera Yasuní. Según los cálculos gubernamentales, dicha explotación «aportará cinco puntos de crecimiento al PIB y ayudará a terminar con la pobreza en 2017» (Caria y Domínguez, 2014, p. 140).

6 El 28 de noviembre de 2012 se oficializó la XI Ronda de licitación petrolera, destinada a la exploración y explotación de trece bloques petroleros en las provincias de Napo, Pastaza, Morona Santiago y Orellana, con incidencia en territorios de siete nacionalidades indígenas, con un traslape en algunos casos de $70 \%$ o $100 \%$ de sus territorios.

7 Conforme expresa Harvey (2014), una clase rentista - para nuestro caso empresas transnacionales petroleras y la estatal petrolera- ejercerían como titulares de derechos de propiedad sobre recursos del subsuelo; monopolizan los recursos con vistas a la extracción de rentas; esto requiere de una naturaleza tornada en mercancía y cosificada. Se explotan recursos energéticos con efectos ecológicos localizados, dejando un paisaje desigual tras de sí — vertederos de residuos tóxicos, ciudades abandonadas o empobrecidas-. Lo que ocurre a la par de la «colonización del mundo de la vida».

8 Con la repotenciación de la planta se ha pretendido procesar 110. ooo barriles de crudo al día. Por su antigüedad y continuas paradas no programadas, estuvo trabajando al $85 \%$ de su capacidad. La estimación competa del costo era de USD 1380 millones, si bien estaba presupuestado en USD 980 millones de dólares. Recuperado de http://www.el comercio.com/actualidad/negocios/rehabilitacion-de-refineriaesmeraldas-cuesta.html.

9 Conforme a datos de Ecuador Estratégico, en la Ciudad del Milenio en Playas de Cuyabeno hay 59 beneficiarios (USD 75.905,76 por cada uno); en Pañacocha 129 beneficiarios con el mismo monto invertido por cada uno. La Ciudad del Milenio habría costado en Playas USD 20.275.468, mientras la de Pañacocha, USD 20.071.117 (Comunidades del Milenio (2013), folleto de PetroAmazonas EPI). 
10 Entre las personas que no tienen ingresos se incluye a todas las personas que no reportan ingresos ni rentas de ningún tipo: básicamente estudiantes, amas de casa, ancianos que no reciben jubilación, personas discapacitadas, desempleadas o trabajadoras familiares sin remuneración.

11 De acuerdo al Informe de Rendición de Cuentas, Ecuador Estratégico (2014), se han invertido en millones de dólares: en la provincia de Sucumbíos 159,2; en Orellana 146,4; en Zamora Chinchipe 121,7; en Napo 102,2; en Morona Santiago 57; y en Pastaza 45,4.

12 El asentamiento de Playas de Cuyabeno tuvo lugar en la década del cincuenta. Desde un inicio tuvo una composición interétnica conformada por familias mestizas ribereñas y kichwas provenientes de la cuenca del río San Miguel, Putumayo, conforme describe Little (2001), si bien la zona era de ocupación originaria de sionas y siekóya pai del Cuyabeno. Conforme las narrativas kichwas, comenzaron a trasladarse por el área desde primeras décadas del xx cuando trabajaban con patrones colombianos durante el término del auge cauchero. En el caso de Pañacocha, el centro poblado pertenece a la parroquia Pañacocha, creada en 1969 en el cantón Shushufindi. Sus primeros habitantes provinieron de una migración en la década del sesenta motivada por misioneros capuchinos que llevaron a familias kichwas de las riberas del río Payamino a trabajar en actividades de ganadería, agricultura y en la construcción de un orfanato. También la habitaron familias colonas provenientes de la Sierra. Actualmente, Playas se compone de familias kichwas en su mayoría, con matrimonios entre kichwas y mestizos de Esmeraldas, Guayaquil e inmigrantes de Colombia. Hay mujeres kichwas casadas con a 'i cofán, aiekóya pai y siona del Cuyabeno. 13 Adicionalmente había el interés de ciertos dirigentes por participar en la economía petrolera y conformar una empresa indígena, Sacha Petrol, lo que no cuajó posteriormente.

14 La tasa de participación laboral global a nivel país es de $53 \%$, y se calcula para las personas de 10 años de edad y más. Es el porcentaje que resulta del cociente entre la población económicamente activa (PEA) y la población en edad de trabajar (РET).

15 Se trata de un hotel construido décadas antes por los misioneros capuchinos para el funcionamiento de un orfanato, que después fue adquirido para funcionar como hotel. Éste ha proporcionado por años alojamiento para personal de empresas petroleras que trabajan en zonas de Sucumbíos aledañas a Pañacocha.

16 Esto dio continuidad en cierta forma a experiencias previas cuando hombres y mujeres se emplearon en actividades de mantenimiento del hotel - antes orfanato y luego adquirido por un empresario colombiano-, y que albergó a operarios petroleros. En éste, varios comuneros, hombres y mujeres, se empleaban en actividades de mantenimiento, limpieza y lavado de ropa.

\section{REFERENCIAS}

Acosta, A., Almeida, A., y Balseca, M. (2011). El Ecuador postpetrolero. Quito, Ecuador: ILDIs.

Acosta, A. (2013). A modo de prólogo. El correísmo: Un nuevo modelo de dominación burguesa. En F. J. Álvarez, et al., El correísmo al desnudo (pp. 9-21), Quito, Ecuador: Montecristi Vive.

Caria, S. y Domínguez, R. (2014). El porvenir de una ilusión: la ideología Del Buen Vivir. En América Latina Hoy, (pp. 139-163). Salamanca, España: Ediciones Universidad de Salamanca.

Federici, S. (2004). Calibán y la bruja: Mujeres, cuerpo y acumulación originaria. Madrid: Traficantes de Sueños Ed.

Harvey, D. (2014). Contradicciones y el fin del capitalismo. Quito, Ecuador: IAEN.

INEC (2006b). Encuesta Nacional de Ingresos y Gastos de los Hogares Urbanos y Rurales. Quito: INEC.

Lander, E. (2013). Tensiones/contradicciones en torno al extractivismo en los procesos de cambio: Bolivia, Ecuador y Venezuela. En C. Arzeet (Comp.) et al., Promesas en su laberinto: cambios y continuidades en los gobiernos progresistas de América Latina. Quito, Ecuador: IEE.

Schminck, M. y Wood, Ch. (1987). The political Ecology of Amazonia. En P. Little y M. Horowitx (Eds.), Lands at risk. Boulder, USA: Westview Press. 
Turner, T. y Brownhill, L. (2006). Ecofeminism as gendered, ethnicized class struggle: A rejoinder to Stuart Rosewarne. Capitalism Nature Socialism, 17(4), s/p. 
